\title{
Planeamiento estratégico y liderazgo pedagógico del directivo, ejes fundamentales para la educación de calidad
}

\author{
Carlos Mallqui Vitor \\ Camavi777@gmail.com
}

Fernando Emilio Escudero Vílchez

fescudero@silfersystem.com

Doctorado en Gestión Pública y Gobernabilidad en la

Universidad César Vallejo

Lima - Perú

\section{RESUMEN}

El objetivo de esta investigación fue: Determinar cómo influye el planeamiento estratégico y el liderazgo pedagógico de los directivos en el logro de la calidad educativa en las instituciones educativas públicas de Carabayllo, en este asunto está respondiendo a los problemas institucionales descritas. Es de enfoque cuantitativo, tipo explicativo con diseño correlacional causal, se tiene como muestra a 144 docentes de diferentes instituciones públicas, se aplicó el cuestionario para el recojo de datos, y para la prueba de hipótesis se ha utilizado la prueba de regresión logística ordinal; Se llegó a la siguiente conclusión: Se logró a determinar que sí existe influencia significativa del planeamiento estratégico y el liderazgo pedagógico del directivo en el logro de la calidad en las instituciones públicas de Carabayllo ; habiéndose obtenido un walt $=48,552$ y 57,476 y un $\mathrm{p}=0,000$, y un coeficiente de variabilidad de Nagelkerke del 55,5\%.

Palabras clave: planeamiento estratégico; liderazgo directivo; calidad educativa. 


\title{
Strategic planning and pedagogical leadership of the manager are fundamental axes for quality education
}

\begin{abstract}
The objective of this research was: To determine how strategic planning and pedagogical leadership of managers influence the achievement of educational quality in public educational institutions in Carabayllo, 2021, this issue is responding to the institutional problems described. It has a quantitative approach, explanatory type with causal correlational design, the sample is 144 teachers from different public institutions, the questionnaire was applied to collect data, and the ordinal logistic regression test was used for the hypothesis test; The following conclusion was reached: It was possible to determine that there is a significant influence of strategic planning and the pedagogical leadership of the manager in the achievement of quality in the public institutions of Carabayllo 2021; having obtained a walt $=48.552$ and 57.476 and $\mathrm{a} p=0.000$, and $\mathrm{a}$ Nagelkerke coefficient of variability of $55.5 \%$.
\end{abstract}

Keywords: strategic planning; executive leadership; educational quality.

Artículo recibido: 30 noviembre. 2021 Aceptado para publicación: 29 diciembre 2021

Correspondencia: Camavi777@ gmail.com Conflictos de Interés: Ninguna que declarar 


\section{INTRODUCCIÓN}

Es muy importante tocar este tema ya que en la actualidad a nivel mundial se está enfrentando grandes desafíos en relación a la educación ya sea en los países super desarrollados como también en las sociedades y organizaciones nacionales y locales. En estos tiempos el mundo es tecnificado y se debe utilizar bien en favor de una calidad de vida y educación. Sin embargo, siguen existiendo los grandes problemas: Falta del diagnóstico situacional real para identificar los problemas relacionados a la educación. Las políticas públicas no ayudan a mejorar la calidad educativa en todo el país. Precariedad de preparación y capacitación a docentes y directivos. Muchas instituciones educativas no cuentan objetivamente con los documentos de gestión pedagógica: Proyecto Educativo Institucional (PEI), Plan Anual de trabajo (PAT), Reglamento Interno (RI) Manual de Organización y Funciones (MOF) y el Informe de Gestión Anual (IGA).

De acuerdo con lo expuesto, se formula como problema ¿Qué influencia existe entre el planeamiento estratégico, liderazgo pedagógico del directivo en la calidad educativa en las instituciones educativas públicas de Carabayllo?, y los objetivos específicos: ¿Qué influencia existe entre el planeamiento estratégico, liderazgo pedagógico del directivo en la eficiencia en las instituciones educativas públicas de Carabayllo?, ¿Qué influencia existe entre el planeamiento estratégico, liderazgo pedagógico del directivo en la eficacia en las instituciones educativas públicas de Carabayllo?, ¿Qué influencia existe entre el planeamiento estratégico, liderazgo pedagógico del directivo en la funcionalidad en las instituciones educativas públicas de Carabayllo?,

\section{Antecedentes}

Al revisar los antecedentes internacionales con respecto a la problemática descrita en líneas anteriores, ubicamos investigaciones, como Díaz (2020), realizó un estudio con el objetivo de diseñar planes estratégicos para prevenir y restaurar la valoración de la calidad educativa. El liderazgo permitió ten ruan visión estratégica para poder desarrollar acciones en la institución educativa, para el logro de las metas y objetivos en la institución Barba y Delgado (2020) realizaron un estudio con el objetivo de conocer la relación que existe entre el liderazgo pedagógico y gestión escolar en la calidad educativa, Quito. De enfoque cuantitativo, nivel descriptivo y diseño correlacional de corte transversal, una muestra de 374 docentes, se utilizó como instrumentos los cuestionarios válidos y confiables, y concluyó: existe una relación directa, moderada y significativa entre el 
liderazgo pedagógico y la gestión escolar en la calidad educativa de instituciones educativas de Quito, Ecuador. Toledo (2020) realizó un estudio con el objetivo de generar un constructo teórico basado en el liderazgo directivo y el desempeño docente. Con un enfoque cuantitativo, llegando a concluir: el estilo de liderazgo predomina en la gestión del director de la institución educativa en el democrático, con un comportamiento respetuoso y compromiso institucional, y motivación para el fomento de las relaciones interpersonales. Reyes ét al. (2017) realizaron un estudio con el objetivo de conocer el grado de relación del liderazgo directivo y la gestión desde la percepción de los estudiantes. De enfoque cuantitativo, tipo básico, nivel descriptivo, y llegó a la siguiente conclusión: Existe una relación directa, moderada y significativa entre el liderazgo directivo y la gestión desde la percepción de los estudiantes.

De la misma manera, las investigaciones nacionales, como: Meza ét al. (2021) realizaron un estudio con el objetivo de determinar el grado de relación entre los estilos de liderazgo y calidad educativa. Con un enfoque cuantitativo, de método y diseño descriptivo correlacional y llegando a concluir: Existe una relación significativa entre los estilos de liderazgo y la calidad educativa en la institución educativa José Antonio Encinas.

Cruz y Santos (2021) realizaron el estudio, con el objetivo de demostrar como la gestión de los directivos se relaciona con la calidad del servicio educativo; con un enfoque cuantitativo, y llegó a la conclusión que existe una relación significativa entre la calidad educativa mediante una adecuada gestión de parte de los directivos.

Espinoza ét al. (2021) realizó el estudio con el objetivo de determinar la relación entre el liderazgo directivo y la calidad de los aprendizajes. Con un método cuantitativo, llegando a la siguiente conclusión: el liderazgo directivo se relaciona con la mejora de la calidad educativa en los estudiantes, con una correlación de 0,94. Vergara ét al. (2021) realizaron un estudio con el objetivo de determinar la relación entre la gestión institucional y la planificación estratégica, con un enfoque cuantitativo y llegando a concluir: existe una relación directa, alta y significativa entre la gestión institucional y la planificación estratégica en instituciones educativas de Jaén, Cajamarca. Coronel (2019) realizó un estudio con el objetivo de determinar la relación entre la gestión educativa y la eficiencia y eficacia en los estudiantes; de enfoque cuantitativo, llegando a la siguiente conclusión: La gestión educativa se relaciona de manera significativa con la eficiente y la eficacia 
educativa en los estudiantes, con un coeficiente de correlación de Spearman $=0,822$ y un nivel de significancia $=0,000$.

También, León (2018) realizó un estudio con el objetivo de determinar el nivel de la calidad educativa; con un enfoque cuantitativo, de tipo básico y diseño no experimental, descriptivo simple, se aplicó un cuestionario con una muestra probabilística de 102 docentes, y llegó a concluir: La dimensión eficacia de la calidad educativa, el 14,0\% indicaron que están en nivel malo, el 57,0\% en nivel regular y el 29,0\% un nivel bueno, con los indicadores nivel de cumplimiento y desarrollo de potencialidades; y con respecto a la eficiencia, el 37,0\% indicaron que tiene un nivel malo, el 51,0\% tiene un nivel regular, y el $12,0 \%$ un buen nivel y con indicadores como aseguramiento de los procesos y prevención de conflictos. Siguiendo la planificación estratégica puede ser definida por varios autores de la siguiente manera, Chiavenato (1998) define la planificación estratégica como un procedimiento de adaptación institucional organizativa de toma de decisiones y evaluación real de la misma y es una herramienta que sirve para subsanar situaciones adversas con la mirada prospectiva en el logro de las metas de desempeño conforme a los procesos planificados.

Sallenave, (2002) define la planificación estratégica como procedimiento en donde los líderes direccionan las metas y sus operaciones en un determinado tiempo y además se puede considerar como un actuar de intercambio para tomar elección conforme a los objetivos de una organización. Rodríguez, Gómez, Mena, \& Loor (2019) en un estudio realizado, señalaron la importancia al modelo de la planificación estratégica para contribuir en la construcción de la sostenibilidad de las organizaciones institucionales que se proyectan al logro de las metas propuestas de muchos éxitos.

En cuanto se refiere al liderazgo pedagógico del director podemos mencionar a los autores que abordaron este tema, García (2018) expresa cuando se habla del liderazgo se entiende principalmente de la capacidad tienen las personas para influir hacia otros. Santos (2018) en su tesis doctoral quiso demostrar si existe la relación o no entre el liderazgo directivo y la prestación de servicio del padre de familia en la búsqueda del mejoramiento de la calidad de enseñanza en el colegio Solertia de Pucallpa y llegó a la conclusión que sí existe significativamente esa relación. Changanaqui \& Huapaya (2017), realizaron su tesis doctoral sobre las formas del liderazgo que mantienen los directivos durante su gestión buscando la calidad educativa, llegó a la conclusión señalando que los docentes 
manifiestan que el liderazgo que maneja el director es de tipo transformacional. Álvarez, Torres, \& Chaparro (2016), manifestaron el liderazgo es una condición indispensable para fortalecer los recursos humanos en un sector empresarial. Gonzales \& López (2020) hicieron estudio sobre la función del liderazgo de los directores evidenciando desde el punto de vista de los interesados, siendo de mayor interés para los directivos en las diferentes dimensiones; y se identifica que en España aún existe un liderazgo tradicional desde el frente, que hasta suena exclusiva del directivo Ritacco \& Amores (2019) señalan el liderazgo pedagógico es una capacidad de integración que impulsa hacia las prácticas educativas de gran valor haciendo diversas gestiones para efectuar las metas propuestas en una organización (p.378).

Cuando se habla de la calidad educativa también debemos considerar los estudios que hicieron los investigadores para comprender el sentido y las metas hacia dónde se dirige. Khalaf, (2021) en el artículo titulado "the role of knowledge management strategies in emproving the quality of educational service", dice sobre la mejora de la educación de calidad, es muy fundamental considerar el conocimiento organizacional y los intercambios de ideas y acciones que se debe mantener entre todos los componentes de la institución educativa sin hacer ninguna discriminación, sólo así se puede conseguir la calidad educativa. Fernandez et al,(2020) manifiestarin que, la valoración la formación de emociones, la empatía y resolución de problemas para dar mayor eficacia en las enseñanzas y aprendizaje en los estudiantes desde la edad de inicial. Todo ello permite evolucionar en la capacidad de estudio para la adquisición de calidad de aprendizajes.

Espinosa \& Prieto (2020) en su artículo concluyen que la calidad educativa se orienta sobre todo a la formación integral que es elemento fundamental de todas las instituciones educativas. Cáceres (2016) dice la calidad educativa depende de los directores y los docentes ya que ellos son los promotores de promover los servicios necesarios para su buen desarrollo, por lo tanto, deben dedicar su compromiso de una manera activa en forma personal y colectiva. Da Fonseca ét al. (2021) manifiesta en su artículo haciendo comparación de sus trayectorias de las reformas educativas en las últimas décadas en relación a la calidad educativa.

CEPLAN, (2016) manifestó que, es muy importante tener en cuenta los contenidos y la forma de enseñar a los escolares para medir la calidad educativa, por lo tanto, es fundamental incentivar la calidad de los docentes como agentes del desarrollo educativo. 
Carrillo (2020) consideró una educación de calidad forma mejores seres humanos, ciudadanos de valor ético, que conocen sus derechos y deberes y conviven en paz.Lo mismo, Reyes, Díaz, Dueñas y Bernal (2016), afirmaron que, la educación de calidad permite al ser humano formarse en calidad, y participar en la construcción de una sociedad de calidad en donde todos los ciudadanos gocen de una vida de calidad.

En referencia a la calidad educativa, el Ministerio de Educación (2018), de acuerdo al Instituto Peruano de Evaluación y Acreditación de la Calidad de la Educación-IPEBA, señaló las siguientes dimensiones: La eficacia educativa, es la medición del logro de los objetivos educativos, que es garantizada por el Estado, como un derecho equitativo, distribuyendo el aprendizaje relevante e importante, asimismo. La dimensión eficacia educativa, es aquella que logra medir el cumplimiento de los objetivos pedagógicos, partiendo de las operaciones, coordinado y realizando actividades ejercidas por el director. Para Condor (2019), es el resultado de un trabajo conjunto, en donde el aprendizaje es logrado por los estudiantes sea óptimo. De la misma manera, Santos (2012) señaló que la eficacia educativa, da cuenta en qué nivel y en qué medida los estudiantes tienen acceso y permanencia en la institución educativa. La eficiencia educativa, para Cóndor (2019) es el grado nivel que se logra al cumplir los objetivos y metas pedagógicas propuestas por la institución educativa, en la utilidad óptima del recurso y medio que dispone el sistema educativo peruano.

\section{ESTRATEGIAS METODOLÓGICAS O MATERIALES Y MÉTODOS}

Este trabajo es de enfoque cuantitativo, que de acuerdo a Naupas, ét al. (2013), está caracterizado por la utilización de procedimiento y técnicas cuantitativas medibles, uso de volumen, observación relacionados a la medición de las unidades de análisis y lo mismo el tratamiento estadístico.

\subsection{Tipo y diseño de investigación}

La investigación fue de tipo básico, que de acuerdo Sánchez, Reyes, y Mejía (2018), permitió el incremento del conocimiento acerca de las relaciones entre las variables, no será de carácter práctico.

El nivel de investigación fue explicativo, donde Carrasco (2019) señaló que permite conocer los factores por los cuales suceden los hechos o fenómenos, teniendo en cuenta las causas y efecto existente en un contexto determinado. 
Así también, fue de diseño correlacional causal, que según Hernández, Fernández y Baptista (2014), determinó el grado de relación de causa-efecto entre las variables independientes planeamiento estratégico y liderazgo pedagógico en la variable dependiente calidad educativa, y responde al siguiente diseño:

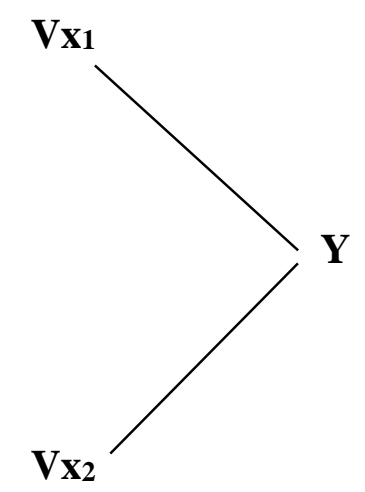

\section{Dónde:}
$\mathrm{Vx}_{1} \quad$ : Planeamiento estratégico
$\mathrm{Vx}_{2}:$ Liderazgo pedagógico
Y : Calidad educativa

\subsection{Variables y operacionalización}

\section{Variable independiente 1 (X): Planeamiento estratégico}

Definición conceptual. Plan de acción y herramienta principal para dar viabilidad y crecimiento de una organización en un plazo determinado con el propósito de establecer cambio de una situación actual a una deseada (Solórzano ét al., 2015).

\section{Variable independiente $2\left(\mathrm{X}_{2}\right)$ : Liderazgo pedagógico}

Definición conceptual. capacidad de integración que impulsa hacia las prácticas educativas de gran valor haciendo diversas gestiones para efectuar las metas propuestas en una organización (Ritacco ét. al, 2019).

\section{Variable dependiente (Y): Calidad educativa}

Definición conceptual. Generación del desarrollo de toda capacidad en el estudiante con una visión amplia en sus logros intelectuales, emocionales, éticos y morales, considerando su nivel socioeconómico, su entorno familiar y sus conocimientos previos (Mortimer, 2008).

\subsection{Población, muestra, muestreo y unidad de análisis}

La población de acuerdo a Arias (2012), es un agrupamiento determinado de componentes que tienen sus características comunes que serán extensivas conclusiones de estudio, que 
quedará determinada por los problemas y objetivos de la investigación, y comprende 144 docentes de las Instituciones educativas públicas de Carabayllo, 2021

Así también la muestra, que según Palella y Martins (2008), es una pequeña parte del conjunto de la población en estudio que poseen rasgos comunes de la manera más exacta posible, pudiendo ser probabilística o no probabilística.

De la misma manera, Canal (2006) señaló que el muestreo, son procedimientos estadísticos que persiguen seleccionar muestras representativas, de donde se piensa conseguir los resultados, con un margen de error determinado. Así mismo, el muestreo fue no probabilístico y censal; está comprendido por la totalidad de la población.

\subsection{Técnicas e instrumentos de recolección de datos}

En la recolección de datos, se utilizó la técnica encuesta, según Tamayo y Tamayo (2012), permitirá recoge la información de manera directa mediante interrogantes, que serán formuladas por la investigadora a los integrantes de la muestra.

De la misma manera, el instrumento seleccionado fue el cuestionario, que de acuerdo a Ander-Egg (2011), consiste en la formulación sistemática de preguntas relacionadas a las hipótesis, así como la verificación de las hipótesis.

La validez del instrumento según Escobar y Cuervo (2008) implica la opinión de expertos referentes al estudio que se realiza, y que tiene reconocimiento de los demás, que evidencian y realizan juicios y valoraciones en criterios como pertinencia, claridad y relevancia.

Asimismo, la confiabilidad del instrumento, que según Hurtado (2010), es el grado de similitud de las respuestas de las observaciones entre el contexto de la investigadora y los integrantes de la muestra; y por ser politómicos se utilizó el coeficiente Alfa de Cronbach, que se obtuvo de la aplicación de una prueba piloto a 20 docentes.

\subsection{Procedimientos}

En el proceso de investigación, se procedió inicialmente a la recopilación de la información, para consignar datos teóricos, luego se solicitó la autorización a las autoridades educativas para la aplicación de los instrumentos. Se aplicaron cuestionarios, para luego recoger los datos fueron procesados en una base de datos en Excel, y luego se procesaron en SPSS para la obtención de los resultados descriptivos e inferenciales. 


\subsection{Método de análisis de datos}

Para el análisis de datos, se utilizó el Excel y el SPSS v25, y fue en dos aspectos: análisis descriptivo, que implicó elaborar tablas y figuras estadísticas y luego el análisis inferencial, que implicó la realización de la prueba de normalidad que por ser la muestra mayor a 50 se utilizó la prueba de Kolmogorov-Smirnov; y en función de los resultados, se utilizó el estadístico para comprobación de las hipótesis.

\subsection{Aspectos éticos}

La investigación estuvo enmarcada en la guía de metodología que propuso la Universidad César Vallejo, en las normas APA en su sétima edición, que permitió consignar datos y referencias de autores nacionales e internacionales; asimismo, se mantuvo a los integrantes de la muestra anónimos. Se respetaron los resultados obtenidos. De la misma manera, luego de culminar el trabajo, se procedió a determinar el porcentaje de similitud a través del software Turnitin, que evidenció la originalidad del estudio.

\section{RESULTADOS Y DISCUSIÓN}

\subsection{Resultados descriptivos}

\section{Tabla 1}

Distribución de frecuencia de la variable planificación estratégica

\begin{tabular}{ccc}
\hline Niveles & Frecuencia & Porcentaje \\
\hline Bajo & 87 & 60,4 \\
Medio & 54 & 37,5 \\
Alto & 3 & 2,1 \\
Total & 144 & 100,0 \\
\hline
\end{tabular}

Figura 1. Niveles de la variable planeamiento estratégico

Planeamiento estratégico

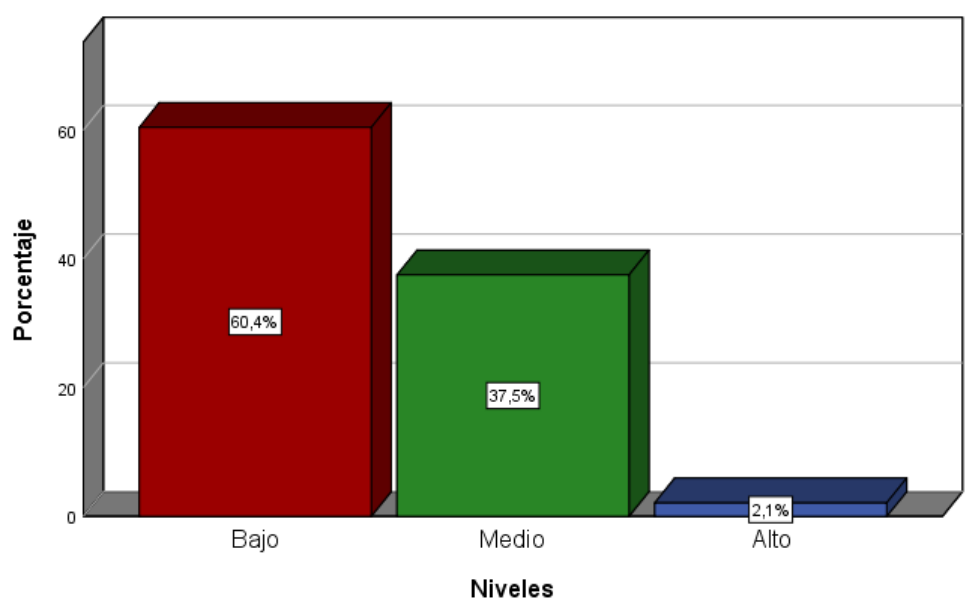


En la figura 1 se observa que, con respecto a la variable planeamiento estratégico, el $60,4 \%$ de los docentes percibió un bajo nivel, el 37,5\% un nivel medio y el 2,1\% un nivel alto, ante ello se puede afirmar que, la mayoría de los docentes percibió un bajo nivel del planeamiento estratégico.

\section{Tabla 2}

Distribución de frecuencia de la variable liderazgo pedagógico

\begin{tabular}{ccc}
\hline Niveles & Frecuencia & Porcentaje \\
\hline Bajo & 101 & 70,1 \\
Medio & 41 & 28,5 \\
Alto & 2 & 1,4 \\
Total & 144 & 100,0 \\
\hline
\end{tabular}

Figura 2. Niveles de la variable liderazgo pedagógico

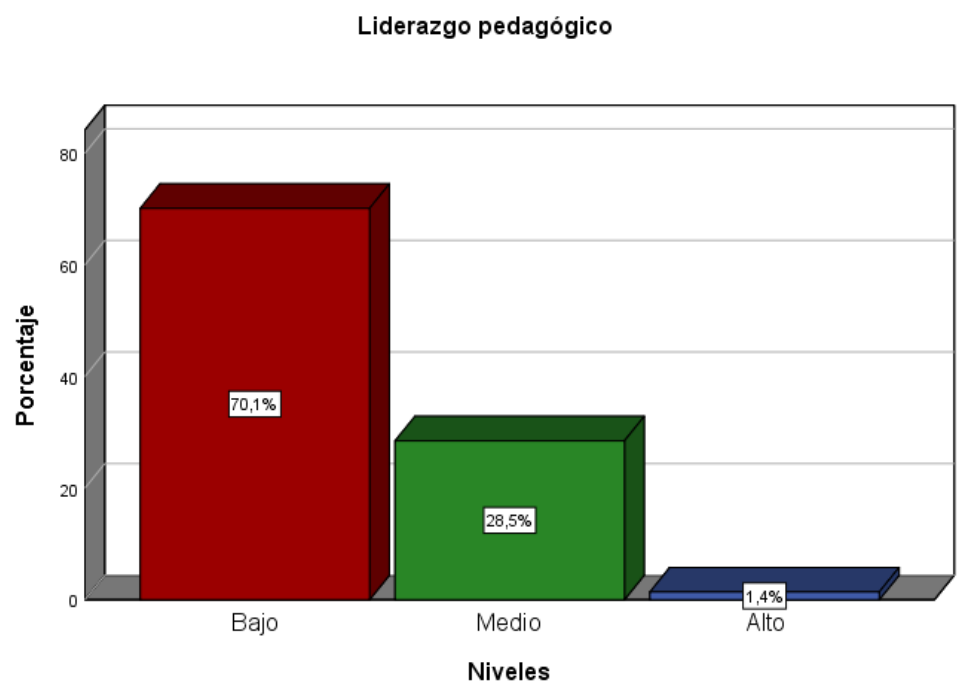

Los resultados de la figura 2 permite observar que, con respecto a la variable liderazgo pedagógico, el 70,1\% de los docentes percibió un bajo nivel, el 28,5\% un nivel medio y el 1,4\% un nivel alto, ante ello se puede afirmar que, la mayoría de los docentes percibió un bajo nivel de liderazgo pedagógico.

\section{Tabla 3}

Distribución de frecuencia de la variable calidad educativa

\begin{tabular}{ccc}
\hline Niveles & Frecuencia & Porcentaje \\
\hline Bajo & 86 & 59,7 \\
Medio & 55 & 38,2 \\
Alto & 3 & 2,1 \\
Total & 144 & 100,0 \\
\hline
\end{tabular}


Figura 3. Niveles de la variable calidad educativa

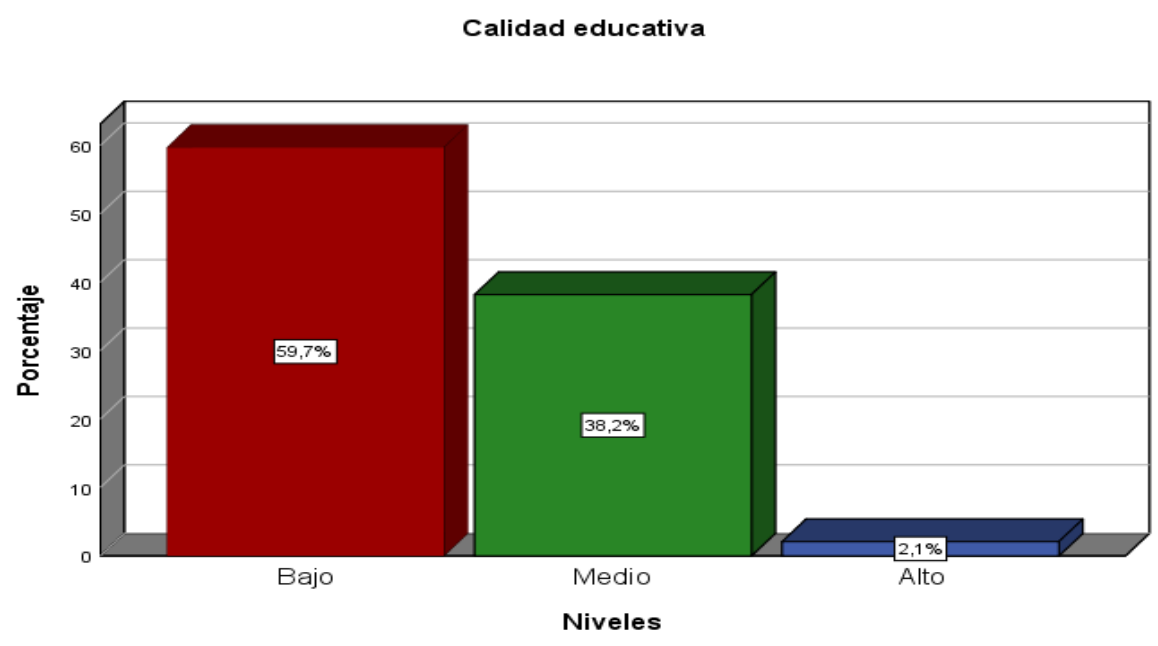

En la figura 3 se observa que, con respecto a la variable calidad educativa, el 59,7\% de los docentes presentó un bajo nivel, el 38,2\% un nivel medio y el 2,1\% un nivel alto, ante ello se puede afirmar que, la mayoría de los docentes presentó un bajo nivel de calidad educativa

\subsection{Resultados inferenciales}

\subsubsection{Prueba de hipótesis}

\section{Hipótesis general}

Tabla 4 Información del ajuste del modelo -Hipótesis general

\begin{tabular}{lcccccc}
\hline Modelo & \multicolumn{3}{c}{ Criterio de ajuste del modelo } & \multicolumn{3}{c}{$\begin{array}{c}\text { Contrastes de la razón de } \\
\text { verosimilitud }\end{array}$} \\
& AIC & BIC & -2 log verosimilitud & Chi-cuadrado & gl & Sig. \\
\hline $\begin{array}{l}\text { Sólo la } \\
\text { intersección }\end{array}$ & 232,315 & 238,255 & 107,348 & & & \\
Final & 232,401 & 240,255 & 17,285 & 90,063 & 8 &, 000 \\
\hline
\end{tabular}

Tabla 5 Pseudo R cuadrado - Hipótesis general

\begin{tabular}{lc}
\hline \multicolumn{1}{c}{ Estadístico } & Valor \\
\hline Cox y Snell &, 432 \\
Nagelkerke &, 555 \\
McFadde &, 375 \\
\hline
\end{tabular}

Función de enlace: Logit.

Con $\mathrm{X}^{2}=90,063, \mathrm{gl}=8$ y $\mathrm{p}=0.000$; el valor de $\mathrm{p}$ ( $\operatorname{sig}$ ) fue menor que $0,05(0,000<0,05)$ entonces se rechaza la hipótesis nula $\mathrm{H}_{0}$ y se acepta la hipótesis planteada por el 
investigador $\mathrm{H}_{1}$ : Existe influencia significativa del planeamiento estratégico y el liderazgo pedagógico del directivo en la calidad educativa en las instituciones educativas públicas de Carabayllo 2021 ; asimismo, al tener el valor de 0,555 en el Pseudo Rcuadrado de Nagelkerke, el mismo que se encuentra cercano al 1, afirmamos que existe una influencia muy alta de las variables planeamiento estratégico y liderazgo pedagógico en la calidad educativa.

\section{Tabla 6}

Estimaciones de parámetro - Hipótesis general

\begin{tabular}{llcccccccc}
\hline & & & & & & \multicolumn{3}{c}{ Intervalo de confianza al } \\
\cline { 6 - 9 } & & Estimación & $\begin{array}{c}\text { Desv. } \\
\text { Error }\end{array}$ & Wald & gl & Sig. & \multicolumn{2}{c}{$\begin{array}{c}\text { Límite } \\
\text { inferior }\end{array}$} & $\begin{array}{c}\text { Límite } \\
\text { superior }\end{array}$ \\
\hline Umbral & {$[$ Cali1 = 1] } & 6,870 &, 986 & 48,552 & 1 &, 000 & 4,937 & 8,802 \\
& {$[$ Cali1 = 2] } & 12,635 & 1,667 & 57,476 & 1 &, 000 & 9,368 & 15,901 \\
Ubicación & Pla1 & 1,759 &, 429 & 16,807 & 1 &, 000 &, 918 & 2,600 \\
& Lide1 & 3,027 &, 503 & 36,288 & 1 &, 000 & 2,042 & 4,012 \\
\hline
\end{tabular}

Función de enlace: Logit.

En la tabla 6 se observa que, la prueba de regresión ordinal con wald mayores que 4, como 48,552 y 57,476, y son adecuados con valores de sig. $0,000<, 05$, en los niveles bajo y medio de la calidad educativa; por ello, se rechaza la hipótesis nula evidenciando que existe una influencia significativa del planeamiento estratégico y el liderazgo pedagógico en la calidad educativa.

\section{Hipótesis específica 1}

\section{Tabla 7}

Información del ajuste del modelo - hipótesis específica 1

\begin{tabular}{|c|c|c|c|c|c|c|}
\hline \multirow[t]{2}{*}{ Modelo } & \multicolumn{3}{|c|}{ Criterio de ajuste del modelo } & \multicolumn{3}{|c|}{$\begin{array}{c}\text { Contrastes de la razón de } \\
\text { verosimilitud }\end{array}$} \\
\hline & AIC & BIC & $-2 \log$ verosimilitud & $\begin{array}{c}\text { Chi- } \\
\text { cuadrado }\end{array}$ & gl & Sig. \\
\hline $\begin{array}{l}\text { Sólo la } \\
\text { intersección }\end{array}$ & 225,372 & 231,312 & 67,644 & & & \\
\hline Final & 225,457 & 233,312 & 20,258 & 47,386 & 8 & 000 \\
\hline
\end{tabular}




\section{Tabla 8}

Pseudo R cuadrado - Hipótesis específica 1

\begin{tabular}{lc}
\hline \multicolumn{1}{c}{ Estadístico } & Valor \\
\hline Cox y Snell &, 195 \\
Nagelkerke &, 255 \\
McFadde &, 150 \\
\hline
\end{tabular}

Función de enlace: Logit.

Con $\mathrm{X}^{2}=47,386, \mathrm{gl}=8 \mathrm{y} \mathrm{p}=0.000$; el valor de $\mathrm{p}(\mathrm{sig})$ fue menor que $0,05(0,000<0,05)$ entonces se rechaza la hipótesis nula $\mathrm{H}_{0}$ y se acepta la hipótesis planteada por el investigador $\mathrm{H}_{1}$ : Existe influencia significativa del planeamiento estratégico y el liderazgo pedagógico del directivo en la eficacia en las instituciones educativas públicas de Carabayllo 2021; asimismo, al tener el valor de 0,255 en el Pseudo R-cuadrado de Nagelkerke, el mismo que se encuentra cercano al 1, afirmamos que existe una influencia muy alta de las variables planeamiento estratégico y liderazgo pedagógico en la dimensión eficacia de la calidad educativa.

\section{Tabla 9}

Estimaciones de parámetro - Hipótesis específica 1

\begin{tabular}{llllllllll}
\hline & & & & & & \multicolumn{3}{c}{ Intervalo de confianza al } \\
& & Estimación & $\begin{array}{c}\text { Desv. } \\
\text { Error }\end{array}$ & Wald & gl & Sig. & \multicolumn{2}{c}{$\begin{array}{c}\text { Límite } \\
\text { inferior }\end{array}$} & $\begin{array}{c}\text { Límite } \\
\text { superior }\end{array}$ \\
\hline \multirow{2}{*}{ Umbral } & {$[$ Efica1 $=1]$} & 3,971 &, 702 & 32,001 & 1 &, 000 & 2,595 & 5,347 \\
& {$[$ Efica1 $=2]$} & 7,746 & 1,066 & 52,839 & 1 &, 000 & 5,657 & 9,835 \\
\multirow{2}{*}{ Ubicaciónn } & Pla1 &, 585 &, 366 & 2,559 & 1 &, 110 &,- 132 & 1,302 \\
& Lide1 & 1,835 &, 392 & 21,940 & 1 &, 000 & 1,067 & 2,603 \\
\hline
\end{tabular}

Función de enlace: Logit.

En la tabla 9 se observa que, la prueba de regresión ordinal con wald mayores que 4, como 32,001 y 52,839, y son adecuados con valores de sig. 0,000 <,05, en los niveles bajo y medio de la dimensión eficacia de la calidad educativa; por ello, se rechaza la hipótesis nula evidenciando que existe una influencia significativa del planeamiento estratégico y el liderazgo pedagógico en la dimensión eficacia de la calidad educativa. 


\section{Hipótesis específica 2}

\section{Tabla 10}

Información del ajuste del modelo - Hipótesis específica 2

\begin{tabular}{lcccccc}
\hline \multirow{2}{*}{ Modelo } & \multicolumn{3}{c}{ Criterio de ajuste del modelo } & \multicolumn{3}{c}{ Contrastes de la razón de } \\
& AIC & BIC & $\mathbf{- 2}$ log verosimilitud & Chi-cuadrado & gl & Sig. \\
\hline $\begin{array}{l}\text { Sólo la } \\
\text { intersección }\end{array}$ & 213,028 & 218,968 & 79,393 & & & \\
Final & 213,113 & 220,968 & 16,548 & 62,845 & 8 &, 000 \\
\hline
\end{tabular}

\section{Tabla 11}

Pseudo R cuadrado - Hipótesis específica 2

\begin{tabular}{lc}
\multicolumn{1}{c}{ Estadístico } & Valor \\
\hline Cox y Snell &, 338 \\
Nagelkerke &, 453 \\
McFadde &, 301 \\
\hline
\end{tabular}

Función de enlace: Logit.

Con $\mathrm{X}^{2}=62,845, \mathrm{gl}=8 \mathrm{y} \mathrm{p}=0.000 ;$ el valor de $\mathrm{p}(\mathrm{sig})$ fue menor que $0,05(0,000<0,05)$ entonces se rechaza la hipótesis nula $\mathrm{H}_{0}$ y se acepta la hipótesis planteada por el investigador $\mathrm{H}_{1}$ : Existe influencia significativa del planeamiento estratégico, liderazgo pedagógico del directivo en la eficiencia en las instituciones educativas públicas de Carabayllo 2021; asimismo, al tener el valor de 0,453 en el Pseudo R-cuadrado de Nagelkerke, el mismo que se encuentra cercano al 1, afirmamos que existe una influencia muy alta de las variables planeamiento estratégico y liderazgo pedagógico en dimensión eficiencia de la calidad educativa.

\section{Tabla 12}

Estimaciones de parámetro - Hipótesis específica 2

\begin{tabular}{|c|c|c|c|c|c|c|c|c|}
\hline & & Estimación & Desv. & Wald & & & Intervalo & $\begin{array}{l}\text { confianza al } \\
\end{array}$ \\
\hline & & Estimacion & Error & Wald & g1 & Sig. & $\begin{array}{l}\text { Límite } \\
\text { inferior }\end{array}$ & $\begin{array}{c}\text { Límite } \\
\text { superior }\end{array}$ \\
\hline Umbral & {$[$ Efic11 = 1] } & 6,109 & ,896 & 46,443 & 1 & ,000 & 4,352 & 7,865 \\
\hline Umoral & {$[$ Efic11 = 2] } & 11,211 & 1,574 & 50,733 & 1 & ,000 & 8,126 & 14,295 \\
\hline Uhicoción & Pla1 & 1,259 & ,408 & 9,523 & 1 & ,002 & ,459 & 2,059 \\
\hline Sorto & Lide1 & 2,563 & ,437 & 34,385 & 1 & , 000 & 1,707 & 3,420 \\
\hline
\end{tabular}

Función de enlace: Logit. 
En la tabla 12 se observa que, la prueba de regresión ordinal con wald mayores que 4, como 46,443 y 50,733, y son adecuados con valores de sig. $0,000<, 05$, en los niveles bajo y medio de la dimensión eficiencia de la calidad educativa; por ello, se rechaza la hipótesis nula evidenciando que existe una influencia significativa del planeamiento estratégico y el liderazgo pedagógico en la dimensión eficiencia de la calidad educativa.

\section{Hipótesis específica 3}

\section{Tabla 13}

Información del ajuste del modelo - Hipótesis específica 3

\begin{tabular}{lcccccc}
\hline \multirow{2}{*}{ Modelo } & \multicolumn{3}{c}{ Criterio de ajuste del modelo } & \multicolumn{3}{c}{ Contrastes de la razón de } \\
& AIC & BIC & -2 log verosimilitud & Chi-cuadrado & gl & Sig. \\
\hline $\begin{array}{l}\text { Sólo } \\
\text { la }\end{array}$ & 231,536 & 237,476 & 82,537 & & & \\
intersección & & & & & & \\
Final & 231,621 & 239,476 & 20,879 & 61,658 & 8 &, 000 \\
\hline
\end{tabular}

\section{Tabla 14}

Pseudo R cuadrado - Hipótesis específica 3

\begin{tabular}{lc}
\hline \multicolumn{1}{c}{ Estadístico } & Valor \\
\hline Cox y Snell &, 320 \\
Nagelkerke &, 418 \\
McFadde &, 266 \\
\hline
\end{tabular}

Función de enlace: Logit.

Con $\mathrm{X}^{2}=61,658, \mathrm{gl}=8 \mathrm{y} \mathrm{p}=0.000$; el valor de $\mathrm{p}(\mathrm{sig})$ fue menor que $0,05(0,000<0,05)$ entonces se rechaza la hipótesis nula $\mathrm{H}_{0}$ y se acepta la hipótesis planteada por el investigador $\mathrm{H}_{1}$ : Existe influencia significativa del planeamiento estratégico, liderazgo pedagógico del directivo en la funcionalidad en las instituciones educativas públicas de Carabayllo 2021; asimismo, al tener el valor de 0,453 en el Pseudo R-cuadrado de Nagelkerke, el mismo que se encuentra cercano al 1, afirmamos que existe una influencia muy alta de las variables planeamiento estratégico y liderazgo pedagógico en dimensión funcionalidad de la calidad educativa. 


\section{Tabla 15}

Estimaciones de parámetro - Hipótesis específica 3

\begin{tabular}{cccccccccc}
\hline & & & & & & & \multicolumn{3}{c}{$\begin{array}{c}\text { Intervalo de confianza } \\
\text { al 95\% }\end{array}$} \\
\cline { 7 - 9 } & & Estimación & $\begin{array}{c}\text { Desv. } \\
\text { Error }\end{array}$ & Wald & gl & Sig. & \multicolumn{2}{c}{$\begin{array}{c}\text { Límite } \\
\text { inferior }\end{array}$} & $\begin{array}{c}\text { Límite } \\
\text { superior }\end{array}$ \\
\hline \multirow{2}{*}{ Umbral } & [Funci1 = 1] & 5,879 &, 868 & 45,886 & 1 &, 000 & 4,178 & 7,580 \\
& {$[$ Funci1 = 2] } & 9,866 & 1,264 & 60,883 & 1 &, 000 & 7,388 & 12,345 \\
\multirow{2}{*}{ Ubicación } & Pla1 & 1,550 &, 396 & 15,282 & 1 &, 000 &, 773 & 2,327 \\
& Lide1 & 2,119 &, 420 & 25,425 & 1 &, 000 & 1,295 & 2,942 \\
\hline
\end{tabular}

Función de enlace: Logit.

En la tabla 15 se observa que, la prueba de regresión ordinal con wald mayores que 4, como 45,886 y 60,883, y son adecuados con valores de sig. $0,000<, 05$, en los niveles bajo y medio de la dimensión funcionalidad de la calidad educativa; por ello, se rechaza la hipótesis nula evidenciando que existe una influencia significativa del planeamiento estratégico y el liderazgo pedagógico en la dimensión funcionalidad de la calidad educativa.

\section{DISCUSIÓN}

Con respecto a la hipótesis general, se tuvo un resultado en la prueba $X^{2}=90,063$ y un $\mathrm{p}=0,000<0,05$, con un Pseudo R-cuadrado de Nagelkerke=0,555, con un wald mayores que 4 , como 48,552 y 57,476, y son adecuados con valores de sig. $0,000<0,05$, lo que permitió aceptar la hipótesis: sí existe influencia significativa del planeamiento estratégico y el liderazgo pedagógico del directivo en la calidad educativa, y tiene semejanza con los planteado por Camarero y Figueroa (2015) quienes señalaron que, a los directivos, aún falta dar satisfacción a las necesidades referentes a su cargo, y ello requiere una formación continua y adecuada para realizar de una manera óptima sus capacidades de liderazgo, y el estudio de García y Romero (2018), quienes concluyeron que existe una influencia significativa de planeación estratégica y la calidad educativa dentro de los colegios de la parroquia el Batán 2018.

En el mismo sentido, Díaz (2020), quién concluyó que, el liderazgo es un factor muy importante dentro de la dirección de una institución, lo cual permite con una visión estratégica, encaminar a la institución hacia la consecución de los objetivos y metas institucionales trazados; así como el estudio de Barba y Delgado (2020) concluyeron que existe una relación directa, moderada y significativa entre el liderazgo pedagógico y la 
gestión escolar en la calidad educativa de instituciones educativas de Quito, Ecuador; y las investigaciones de Toledo (2020) quien llegó a concluir: el estilo de liderazgo predomina en la gestión del director de la institución educativa en el democrático, con un comportamiento respetuoso y compromiso institucional, y motivación para el fomento de las relaciones interpersonales.

También tiene semejanza con la investigación de Reyes ét al. (2017) quienes concluyeron que existe una relación directa, moderada y significativa entre el liderazgo directivo y la gestión desde la percepción de los estudiantes; y el estudio de Changanaqui \& Huapaya (2017), quienes llegaron a la conclusión señalando que los docentes manifiestan que el liderazgo que maneja el director es de tipo transformacional, sin embargo, en la práctica predomina su carácter correctivo evitando el desarrollo eficiente en la gestión de educación de calidad.; y de Rodríguez (2018) quién señaló que, hablar del liderazgo educativo y desafíos, se puede considerar únicamente asumiendo la participación activa de un liderazgo que sea capaz de construir a través de la negociación, consenso, las estrategias y alternativas en el análisis de las posibilidades constructivas; así como el estudio de Khalaf, (2021) quién señaló que es importantel considerar el conocimiento organizacional y los intercambios de ideas y acciones que se debe mantener entre todos los componentes de la institución educativa sin hacer ninguna discriminación, sólo así se puede conseguir la calidad educativa.

De la misma manera, el estudio de Meza ét al. (2021), quienes concluyeron Existe una relación significativa entre los estilos de liderazgo y la calidad educativa en la institución educativa José Antonio Encinas, y la investigación de Cruz y Santos (2021) quienes llegaron a la conclusión que existe una relación significativa entre la calidad educativa mediante una adecuada gestión de parte de los directivos; también el estudio de Espinoza ét al. (2021) llegando a la conclusión que el liderazgo directivo se relaciona con la mejora de la calidad educativa en los estudiantes, con una correlación de 0,949; y se asemeja al estudio de Vergara ét al. (2021) quienes llegaron a concluir: existe una relación directa, alta y significativa entre la gestión institucional y la planificación estratégica en instituciones educativas de Jaén, Cajamarca.

Ello, también se fundamenta en lo planteado por Cáceres (2016), quién señaló que, la calidad educativa depende de los directores y los docentes ya que ellos son los promotores de promover los servicios necesarios para su buen desarrollo, por lo tanto, deben dedicar 
su compromiso de una manera activa en forma personal y colectiva, y Carrillo (2020), quién consideró la eficiencia de la educación en la formación de ciudadanos con valores éticos, que conocen sus derechos y deberes y conviven en paz. Condor (2019) señaló es el grado nivel que se logra al cumplir los objetivos y metas pedagógicas propuestas por la institución educativa, en una utilización óptima de los recursos y medios con que dispone el sistema educativo peruano; representando de esta manera, la capacidad para lograr el efecto esperado con la realización de las acciones educacionales, mejorando y asegurando el futuro de cada estudiante.

De la misma manera, la hipótesis específica 3 tuvo resultado en la prueba $X^{2}=61,658$ y un $\mathrm{p}=0,000<0,05$, con un Pseudo R-cuadrado de Nagelkerke=0,418, y un wald mayores que 4 , como 45,886 y 60,883, y son adecuados con valores de sig. $0,000<, 05$; lo que permitió aceptar la hipótesis: sí existe influencia significativa del planeamiento estratégico y el liderazgo pedagógico del directivo en la funcionalidad de la calidad educativa, y tiene semejanza con el estudio realizado por Santos (2018), quién concluyó, que existe una relación significativa en la prestación del servicio de los padres en el mejoramiento funcional, y el estudio de Alvarez ét al. (2016) quién señaló la condición indubitable del liderazgo en la gestión estratégica como un elemento funcional de la calidad educativa; así como León (2018) quién tuvo como hallazgo que, con respecto a la eficiencia, el 37,0\% indicaron que tiene un nivel malo, el 51,0\% tiene un nivel regular, y el $12,0 \%$ un buen nivel y con indicadores como aseguramiento de los procesos y prevención de conflictos

De La Orden (2007), señaló que la funcionalidad se identifica con las capacidades de un sistema, o de una institución educativa para lograr el ajuste de los objetivos, y mediante ellos, productos, acciones y medios con los que se dispone, a toda función que se le asigna en la sociedad. Los indicadores de la funcionalidad, plantean dificultades en sociedades pluralistas y democráticas; así, la diversidad de valores, aspiraciones y necesidades sociales que coexisten en ellas, y por otro lado las dificultades sobre las metas educativas propuestas.

\section{CONCLUSIÓN O CONSIDERACIONES FINALES}

\section{Primera}

Se determinó que, existe influencia significativa del planeamiento estratégico, liderazgo pedagógico del directivo en la calidad educativa en las instituciones educativas públicas 
de Carabayllo 2021; habiéndose obtenido un resultado en la prueba $X^{2}=90,063$ y un $\mathrm{p}=0,000<0,05$, con un Pseudo R-cuadrado de Nagelkerke=0,555.

\section{Segunda}

Se determinó que, existe una influencia significativa del planeamiento estratégico, liderazgo pedagógico del directivo en la eficiencia en las instituciones educativas públicas de Carabayllo 2021; habiéndose obtenido un resultado en la prueba $\mathrm{X}^{2}=47,386$ y un $\mathrm{p}=0,000<0,05$, con un Pseudo R-cuadrado de Nagelkerke=0,255.

\section{Tercera}

Se determinó que, existe influencia significativa del planeamiento estratégico, liderazgo pedagógico del directivo en la eficacia en las instituciones educativas públicas de Carabayllo 2021; habiéndose obtenido un resultado en la prueba $\mathrm{X}^{2}=62,845$ y un $\mathrm{p}=0,000<0,05$, con un Pseudo R-cuadrado de Nagelkerke=0,453.

\section{Cuarta}

Se determinó que, existe influencia significativa del planeamiento estratégico, liderazgo pedagógico del directivo en la funcionalidad en las instituciones educativas públicas de Carabayllo 2021, habiéndose obtenido un resultado en la prueba $\mathrm{X}^{2}=61658$ y un $\mathrm{p}=0,000<0,05$, con un Pseudo R-cuadrado de Nagelkerke=0,418.

\section{RECOMENDACIONES}

\section{Primera}

Se recomienda a los directivos y funcionarios de la Ugel, la implementación de programas sobre planeamiento estratégico y liderazgo pedagógico en los directivos para viabilizar la calidad de las actividades de la institución educativa en tiempos de pandemia, y optimizar el servicio educativo, teniendo en cuenta la participación activa de los docentes como elementos funcionales de la calidad de servicio educativo.

\section{Segunda}

Se sugiere la implementación de actividades relacionadas al análisis y formulación de estrategias, así como de actividades de liderazgo gerencias y organizacional, para el logro de las metas y distribución de oportunidad y el servicio que brindan las instituciones educativas sean eficaces.

\section{Tercera}

Se recomienda a los directivos de las instituciones educativas fomentar en los docentes, la participación en la planificación estratégica de la institución educativa, así como el 
reforzamiento de las capacidades de liderazgo en los directivos, que este orientado al logro del aprendizaje; a una adecuada distribución de recursos, con altos niveles de eficiencia del servicio educativo.

\section{Cuarta}

Se sugiere a los directivos y funcionarios de la Ugel de Comas, jurisdicción a la que pertenecen las instituciones educativas, implementar estrategias de orientación y fomentos de planes estratégico y liderazgo pedagógico, que implique brindar respuestas a las necesidades de los estudiantes, ejecutando proyectos educativos tendientes a la formación integral de los estudiantes,

\section{LISTA DE REFERENCIAS}

Álvarez, J., Torres, A., \& Chaparro S, E. (2016). Diagnóstico del liderazgo educativo en las Instituciones

Obtenido

de

file:///C:/Users/usuario/onedrive/escritorio/materiales\%20para\%20e1\%20proyect o/liderazgo\%20educativo.pdf

Barba, L. y Delgado, K. (2020). Gestión escolar y liderazgo del directivo: Aporte para la calidad educativa. School management and management leadership: Contribution to educational quality. Revista Educare Universidad Pedagógica Experimental Libertador, Venezuela. vol. 25, núm. 1. http://portal.amelica.org/ameli/jatsRepo/375/3752262013/index.html.

Barragan C, J. y. (2020). Análisis FODA como elemento de la Planeación estratégica. Daena: International Journal of Good Conscience. 15, 222-229. Obtenido de https://eds.p.ebscohost.com/eds/pdfviewer/pdfviewer?vid=7\&sid=e6e51810654f-4b7a-9647-707d5c21b6be\%40redis

Belloni, E, Leccese F., Salvadori G, Rocca M., Buratti C. (2019). A method to assess lighting quality in educational rooms using analytic hierarchy process. Building and Environment, Volume 168, 15 January. shorturl.at/imzHL.

Cáceres, A. (2016). Incidencia del uso de instrumentos tecnológicos en el mejoramiento de la calidad educativa en la Unidad de Postgrado de la Facultad de Educación de la UNMSM. Universidad Nacional Mayor de San Marcos

Camarero, M. (2015). Dirección escolar y liderazgo: Análisis del desempeño de la figura directiva en centros de educación primaria de Tarragona. Universidad de 
Rónvira,

España.

Obtenido

de

https://www.tdx.cat/bitstream/handle/10803/403206/TESI.pdf?sequence=1

Carrillo, E. (2020). Proyecto pedagógico para elevar la calidad educativa en la Licenciatura en Educación Superior de la Unidad Académica de Ciencias de la Educación de la Universidad Autónoma de Guerrero (UAGro) a partir del nuevo Modelo Educativo 2017 en Chilpancingo, Guerr. Revista Dilemas Contemporáneos: Educación, Política y Valores. Año: VII, Número: Edición Especial, Artículo no.1. Obtenido de shorturl.at/htzV8.

CEPLAN. (2016). La educación del futuro y el futuro de la educación. Recuperado de https://www.ceplan.gob.pe/documentos_/a-educacion-del-futuro-y-el-futuro-dela-educacion/

https://eds.s.ebscohost.com/eds/pdfviewer/pdfviewer?vid=4\&sid=c79d8717-64e4-4065af78-a0066f82309e\%40redis

Changanaqui, M., \& Huapaya , G. (2017). Estilos de liderazgo directivo y la calidad de gestión educativa desde la percepción docente de cuatro instituciones educativas públicas. Universidad Marcelino Champagnat, Perú.

Obtenido de http://repositorio.umch.edu.pe/handle/UMCH/434

Chiavenato, I. (1998). Introducción a la teoría general de la administración. Colombia: McGraw-Hill Interamericana S.A. Obtenido de https://esmirnasite.files.wordpress.com/2017/07/i-admon-chiavenato.pdf

De La Orden, A. (2007). Desarrollo y validación de un modelo de calidad universitaria como base para su evaluación. Relieve, 3 (1)

Espinoza, E.; Ramírez, V.; Vildoso, J. y Berrocal, S. (2021). El liderazgo directivo y la calidad de los aprendizajes estudiantes egresados de las instituciones educativas de la red 13 - UGEL - 04, Comas. The directive leadership and the quality of the learning students graduated from the educational institutions of the network 13 UGEL - 04, Comas. Revista Dilemas Contemporáneos: Educación, Política y Valores. Año: VIII. Número 4 Edición Especial.

Garira, E. (2020). Needs assessment for the development of educational interventions to improve quality of education: A case of Zimbabwean primary schools. Education. Sciences. 10, 175; doi:10.3390/educsci10070175 
Gonzales, R., \& López, E. (2020). Evidence of pedagogical leadership of principals,. Obtenido de shorturl.at/avwAU.

Khalaf, M. (2021). The role of knowledge management strategies in improving the quality of educational service - University of Anbar as a model. Material Today.https://www.sciencedirect.com/science/article/pii/S2214785321034507

Rodríguez, L.; Vior, S., \& Stella, R. (2016). Policies for the Assessment of Educational Quality in Argentina. Educação \& Realidade; Vol 43, No 4. https://seer.ufrgs.br/educacaoerealidade/article/view/84907

León, H. (2016). Gestión de la planificación estratégica para el desarrollo profesional docente. Revista de Gestión de la Educación, Vol. 6, № 1, 2215-2288, enerojunio. https://doi.org/10.15517/rge.v1i1.22719.

Meza, M. ét al. (2021). Executive leadership and educational quality in the educational institution Jose Antonio Encinas UGEL 15. Ciencia latina; Vol 5, No 4. http://dx.doi.org/10.37811/cl_rcm.v5i4.754.

Ñaupas, H., Valdivia, M., Palacios, J., \& Romero, H. (2013). Metodología de la investigación cuantitativa-cualitativa y redacción de la tesis. Bogotá: Ediciones de la U.

Reyes, G., Díaz, G., Dueñas, J., \& Bernal, A. (2016). ¿Educación de calidad o calidad de la educación? Uno de los Objetivos de Desarrollo Sostenible y el camino para el desarrollo humano. Revista de la Universidad de la Salle $n^{\circ} 71$, art. 13 Obtenido de shorturl.at/bfqyJ.

Ritacco, M., \& Amores, F. (2019). Capacidades del liderazgo pedagógico en la dirección escolar de centros ubicados en zonas de riesgo social: un estudio cualitativo. Revista mexicana de investigación educativa. RMIE, vol.24, n.81, pp.375402. Obtenido de shorturl.at/akyKO.

Rodríguez, S., Gómez, L., Mena, S., \& Loor., J. (2019). Strategic planning to improve the quality of the construction of homes of the Diteco Constructores Company, with the Ceibo Real project, in the city of Quevedo. Dilemas contemporáneos. Educación, política y valores; Vol VI, No Especial. Obtenido de shorturl.at/uKMV8. 
Romero, C. (2021). Principal Leadership in schools that overcome contextual barriers. REICE. Revista Iberoamericana sobre Calidad, Eficacia y Cambio en Educación 19(1), 73-90 Obtenido de shorturl.at/dvFP4.

Sallenave, J. (2002). Gerencia y planeación estratégica. Bogotá: Norma.

Santos, M. (2018). Relación entre liderazgo directivo y calidad de servicio en la concepción de los padres de familia en la I.E.P.Sollertia, Pucallpa, Ucayali, 2018. Universidad César Vallejo. Obtenido de shorturl.at/rBKX7.

Toledo, A. (2020). Liderazgo directivo y desempeño docente. Educational leadership and teaching performance. Revista Compás Empresarial, 10(30), 76-99. https://doi.org/10.52428/20758960.v10i30.123.

Vergara, E.; De la Cruz, M.; Vértiz, J.; Fernández, F.; Quispe, D. y Vértiz, R. (2021). Gestión institucional y planificación estratégica en una institución educativa pública del distrito de Jaén, Cajamarca, Perú. Institutional management and strategic planning at a school of the district of Jaen, Cajamarca, Perú. Revista Científica Pakamuros, 9(1), 42-53. https://doi.org/10.37787/pakamurosunj.v9i1.162. 